\title{
Spleen Organoid Units Generate Functional Human and Mouse Tissue-Engineered Spleen in a Murine Model
}

\author{
Kristin Gee, MD,, ${ }^{1, \star}$ Mubina A. Isani, MD,, ${ }^{1, \star}$ Alexa Fode, BS,, ${ }^{1, *}$ Kathryn M. Maselli, MD, \\ Samuel M. Zuber, MD,' Kathryn L. Fowler, MD, Anthony I. Squillaro, MD, \\ Laura-Marie A. Nucho, MS, and Tracy C. Grikscheit, MD ${ }^{1,2}$
}

\begin{abstract}
Introduction: Splenectomy is common after trauma or hematologic disease, and alters immune protection against pathogens, which may lead to fulminant infection with high mortality. Yet the spleen has demonstrable regenerative capacity and cells might be recovered and reimplanted at the time of injury or excision to avoid these risks.

Methods: Tissue-engineered spleen (TESp) was generated from Actin ${ }^{G F P}$ mice (mTESp) or human donor spleen (hTESp) through implantation of spleen organoid units (spleen OU), in NOD/SCID mice with concurrent splenectomy, on a biodegradable scaffold. Explants were evaluated and blood smears were obtained to investigate the presence of target cells or Howell-Jolly bodies, which are erythrocyte sequelae of asplenia.

Results: TESp was generated from mouse (mTESp) and human (hTESp) donor cells with essential splenic components: red and white pulp with trabeculae. mTESp and hTESp demonstrated green fluorescent protein- or lamin-positive costaining with proliferating cell nuclear antigen, CD4, and CD11c, identifying proliferative donor cells and key immune components of the spleen of donor origin. Animals with hTESp and mTESP combined with splenectomy had significantly fewer Howell-Jolly bodies on blood smears than controls.

Conclusion: TESp from mouse and human donor cells can be generated by 4 weeks and contains donor immune cells identified by CD4 and CD11c. TESp reduces postsplenectomy erythrocyte inclusions, indicating possible function.
\end{abstract}

Keywords: spleen, splenectomy, organoid units, adult stem cells, hyposplenism, tissue-engineered spleen

\section{Impact Statement}

Overwhelming postsplenectomy infection is rare but highly mortal. Tissue-engineered spleen (TESp) was generated from murine (mTESp) and human (hTESp) donors and appeared histologically similar to native spleen. Both mTESp and hTESp demonstrated proliferative cells of donor spleen origin. Importantly, functional cells were demonstrated on imaging with a corresponding reduction in the number of erythrocyte inclusions in blood smears that are typically identified in patients with asplenia and indicate a lack of clearance by functional spleen tissue. Taken together, these findings indicate that this approach might be clinically relevant as a future human therapy.

\section{Background}

$\mathbf{H}$ YPOSPLENISM, OR A poorly functioning spleen, and asplenia, or splenic absence, are not uncommon. ${ }^{1}$ The spleen is a central regulator of immune homeostasis and scavenger of damaged red blood cells, and a decline in its function may accompany medical disease. Complete absence is normally a consequence of surgical resection. The spleen is removed for many reasons but, most commonly, splenectomy is required after abdominal trauma because of massive bleeding. Splenectomy, partial or complete, is also performed to control some hematologic diseases, such as hereditary spherocytosis, in which it is desirable to limit the normal destruction of abnormal or senescent red blood cells by the red pulp. ${ }^{2}$

\footnotetext{
${ }^{1}$ Division of Pediatric Surgery and Developmental Biology and Regenerative Medicine, Saban Research Institute, Children's Hospital Los Angeles, Los Angeles, California.

${ }^{2}$ Department of Surgery, Keck School of Medicine, University of Southern California, Los Angeles, California.

*These authors contributed equally to this work.
} 
However, without a functional spleen to clear infection and damaged red blood cells, the incidence of infectious complications ranges from $14 \%$ to $29 \% .^{3}$ Overwhelming postsplenectomy infection (OPSI) results in a mortality rate of up to $50 \%$ and can occur many years after splenectomy has been performed. ${ }^{4,5}$ Even with a low incidence of OPSI, children who have had a splenectomy for either trauma or hematologic disease have a lower quality-adjusted life expectancy. ${ }^{6}$ Pediatric splenectomy patients are more susceptible to pneumonia, wound infections, pancreatitis, and venous thromboembolism. ${ }^{6,7}$

Although splenic salvage is often not indicated in massive traumas in which the spleen must be rapidly removed to stop life-threatening hemorrhage, postsplenectomy risks are important enough to attempt partial splenectomies to salvage function in patients with hematologic disorders. Restoring splenic function by implanting tissue-engineered spleen (TESp) might reduce OPSI and its complications in patients who require splenectomy, however urgent, or to salvage patients with hematologic diseases in whom partial splenectomy was technically difficult.

The spleen is composed primarily of lymphoid tissue, which helps to initiate the immune response to pathogens, ${ }^{1,8}$ and has regions of red and white pulp divided by irregular trabeculae. The red pulp, among other functions, serves to remove damaged erythrocytes, whereas the white pulp organizes immune responses to infection and contains multiple immune cell types, including lymphocytes and dendritic cells. The red and white pulps are separated by a marginal zone in which antigen presentation and sequestration of some pathogens occur. ${ }^{9}$

Heterotopic spleen regeneration, or splenosis, is a wellknown phenomenon and is noted when fragments of spleen remain in the abdomen, either subsequent to trauma or after perturbations during development (in which technically these spleen deposits are denoted accessory spleens). In a small number of cases, spleen regeneration was observed after deliberate introduction as an implanted spleen slice. ${ }^{10}$ However, these early instances of splenosis do not fully prevent the fulminant course of OPSI, possibly because there is inadequate tissue volume or function. ${ }^{1}$

We, therefore, sought some years ago in a pilot study in Lewis rats to generate TESp from syngeneic spleen tissue on a hydrolysable polymer scaffold. The resulting TESp in a small cohort of Lewis rats increased survival of pneumococcal septicemia from $41 \%$ to $85 \%$ as compared with animals without a spleen. ${ }^{10}$ This initial study was based on previous adult stem cell approaches for the gastrointestinal tract in which transplantation of multicellular organoid units (OU) of mesenchymal and epithelial origin from the gastrointestinal tract resulted in growth of tissue-engineered stomach, esophagus, small intestine, and colon that contained all of the functional components of the donor cells. ${ }^{11-17}$ Because the spleen is a solid organ compared with the previous hollow organs in which this technique originated, variations in OU production were required.

Most cases of asplenia result from planned or emergent removal of the spleen, so recovery of spleen OU and either immediate or subsequent transplantation might result in functional TESp with possible future value for preventing OPSI and improving quality-adjusted life expectancy after splenectomy for trauma or hematologic pathology.

\section{Materials and Methods}

All animal protocols were approved by the IACUC. Human tissue was obtained after consent from an IRBapproved protocol.

\section{Generation of spleen OU from mice and humans}

Spleen OU were produced by dissecting spleens from 14to 16-day-old Actin ${ }^{G F P 12}$ mice maintained on a C57BL/6 background or from human spleen by enzymatic digestion and mechanical disruption into multicellular clusters in a variation of a previously published protocol. ${ }^{12}$ Human spleen was obtained from operative specimens of 7-12year-old patients after IRB approval and informed consent. The spleen was washed with Hank's balanced salt solution (HBSS, Cat \#14170-112; Gibco) that contained 1\% antibiotic-antimycotic (A/A, Cat \#15240-062; Gibco). The tissue was cut into $2 \times 2 \mathrm{~mm}$ pieces and placed in HBSS with dispase at $0.3186 \mathrm{U} / \mathrm{mL}$ (Cat \#17105-041; Gibco) and collagenase type 4 (Cat \#LS004197; Worthington) at $800 \mathrm{U} / \mathrm{mL}$ on an orbital shaker at $37^{\circ} \mathrm{C}$ for $20 \mathrm{~min}$. The tissue was triturated with a $25 \mathrm{~mL}$ serological pipette (Cat \#89130-900; VWR) until the first point at which the tissue could be drawn up into a $10 \mathrm{~mL}$ serological pipette. After the tissue easily passed into the $10 \mathrm{~mL}$ serological pipette (Cat \#89130-898; VWR), the solution was combined with a solution containing Dulbecco's modified Eagle's medium (DMEM, Cat \#10829-018; Gibco), 10\% heat-inactivated fetal bovine serum (FBS, Cat \#26140079; Thermofisher), 1\% A/A, and 1\% nonessential amino acid (NEAA, Cat \#11140-050; Thermofisher). The mixture was centrifuged at $129 \mathrm{~g}$ for $5 \mathrm{~min}$ at $4^{\circ} \mathrm{C}$. The supernatant was removed and washed with DMEM, 10\%FBS, AA, and NEAA solution and spun at 129 $g$ for $5 \mathrm{~min}$ at $4^{\circ} \mathrm{C}$. In total, $60 \mu \mathrm{L}$ of pellet was then loaded onto each biodegradable polyglycolic acid-poly-L lactic acid scaffold that was generated with previously published methods ${ }^{12}$ and kept at $4^{\circ} \mathrm{C}$ until implantation, which occurs in $<60$ min. ${ }^{18}$ This volume of OU was extrapolated from previous studies conducted in this laboratory. The same process was carried out to create human spleen OU samples with the exception of a longer trituration process in the $25 \mathrm{~mL}$ pipette, required for the greater tissue density. Once the tissue could pass with difficulty into the $10 \mathrm{~mL}$ pipette, the process was the same as the mouse tissue protocol.

\section{Hematoxylin and eosin staining of human spleen $\mathrm{OU}$}

Before implantation, some of the human spleen was preserved in agarose (Cat \#GR140-500; Denville Scientific, Inc.) and then embedded into paraffin blocks. Then $5 \mu \mathrm{m}$ sections of tissue were cut from the blocks and mounted on slides. Slides were deparaffinized, serially rehydrated, and then dipped into hematoxylin (Cat \#MHS16-500ML; Sigma-Aldrich) for $5 \mathrm{~min}$. Slides were washed clear and then dipped into eosin (Cat \#HT110332-1L; Sigma-Aldrich) for $30 \mathrm{~s}$. Slides were once again washed, dipped into $90 \%$ and then 100\% ethyl alcohol (Cat \#111000200; Pharmco) for $1 \mathrm{~min}$ each and then dried. Slides were mounted in Permount (Cat \#17986-01; Electron Microscopy Science) and coverslips were applied. Images were taken with brightfield microscopy at $40 \times($ Leica DM1000, Buffalo Grove, IL) 


\section{Immunofluorescent staining of human spleen $\mathrm{OU}$}

Paraffin-embedded sections of human spleen OU were deparaffinized in histochoice (Cat \# 97060-934; VWR Life Science) and sequentially rehydrated followed by antigen retrieval with Tris-based antigen unmasking solution ( $\mathrm{pH}$ 9.0) (Cat \#H3301 ; Vector) in microwave at full power for $20 \mathrm{~min}$. Slides were then cooled to room temperature and blocked with $2 \%$ donkey serum in tris buffered saline (TBS, Cat \#TBS03-02; Bioland Scientific) with 0.1\% Tween (TBS-T, Cat \#9005-64-5; Amresco) for $30 \mathrm{~min}$. The slides were incubated for $8 \mathrm{~h}$ at $4^{\circ} \mathrm{C}$ with primary antibody against proliferating cell nuclear antigen (PCNA) at a 1:100 dilution (Cat \#sc-9857; Santa Cruz Biotechnology). Slides were then washed with TBS-T and incubated in secondary antibody indocarbocyanine3 (Cy3, Cat \#712-165-150; Jackson Immunoresearch) at 1:200 for $1 \mathrm{~h}$ at room temperature. Slides were rinsed with TBS-T, mounted in Vectashield with 4',6-diamidino-2-phenylindole (DAPI, Cat \#H-1200; Vector), and then imaged on a fluorescent microscope at $40 \times($ Leica DM1600B $)$.

\section{Implantation of mouse and human spleen $O U$ into NOD/SCID mice}

Four groups of adult NOD.Cg-Prkdc ${ }^{\text {scid } I l 2 ~} \mathrm{rg}^{\mathrm{tm} l \mathrm{Wjl}} / \mathrm{SzJ}$ (NOD/SCID) mice (Cat \#005557; Jackson Laboratory) were created: a wildtype control with no surgical intervention $(n=8)$, mice with splenectomy only $(n=7)$, mice with a splenectomy and implantation of mouse spleen OU $(n=13)$, and mice with a splenectomy and implantation of human spleen OU $(n=20)$. Mice were induced in an anesthesia chamber with isofluorane. The murine abdomen was prepped with sterile solution followed by a midline subxiphoid incision. The stomach was located and retracted medially to find the spleen. The spleen and its attachments and vascular supply were dissected free with cautery and the spleen was removed from the abdomen. For mice with omental implants, the spleen OU loaded scaffold was securely wrapped in omentum and secured with a single 5-0 Monocryl (Cat \#Y493G; Ethicon) stitch. The skin and fascia were closed with a 4-0 Vicryl suture (Cat \#J422H; Ethicon) in a running manner. At 1 month, mice were humanely euthanized, and a cardiac puncture was performed to obtain blood for smears and platelet counts. The scaffold was harvested and preserved in formalin. NOD/SCID mice that had a splenectomy alone (as mentioned) and NOD/SCID mice that had no surgery served as controls for platelet counts and blood smear images. Mice with a splenectomy alone were verified to have no evidence of splenosis, or residual spleen, at the time of harvest.

\section{Hematoxylin and eosin staining of murine tissue- engineered spleen and human tissue-engineered spleen}

Murine tissue-engineered spleen (mTESp) and human tissue-engineered spleen (hTESp) explants were preserved in formalin and embedded in paraffin blocks. Then $5 \mu \mathrm{m}$ sections of tissue were cut from the blocks and mounted on slides. Hematoxylin and eosin staining was performed using the same technique as reported previously.

\section{Platelet counts and blood smear analysis of postsplenectomy mice}

Blood smears were stained by the clinical pathology department of Children's Hospital Los Angeles with a Wright
Giemsa stain on a fully automated machine. The clinical pathology department of Children's Hospital Los Angeles also calculated a complete blood count with differential on a Sysmex XN-3000 automated hematology analyzer (Sysmex, Lincolnshire, IL) from cardiac puncture blood stored in BD heparin-lined Microtainers (Cat \#827589N; BD Microtainer). Images from the blood smear were obtained on a Zeiss Axioplan microscope with Diagnostic Instruments SPOT Insight $Q E$ color CCD camera at $63 \times$ magnification with oil immersion (Zeiss, Germany). Blood smears were analyzed for Howell-Jolly bodies and target cells by a blinded trained observer. These are erythrocyte inclusions that result as a sequela of splenectomy because they are no longer cleared by the absent spleen. Cells were counted as number per high-power field (hpf) with $20 \mathrm{hpf}$ analyzed per mouse. Statistical analysis was performed as mentioned hereunder.

\section{Immunofluorescence staining of mTESp and hTESp}

The paraffin-embedded sections of both hTESp and mTESp were deparaffinized in histochoice (Cat \# 97060934; VWR Life Science) and sequentially rehydrated followed by antigen retrieval with $10 \mathrm{mM}$ sodium citrate $(\mathrm{pH}$ 6.0) (Cat \#H-3300; Vector) in the microwave at half power. For CD4 and CD11c staining, deparaffinization and serial rehydration were followed by antigen retrieval with Trisbased antigen unmasking solution ( $\mathrm{pH} \mathrm{9.0)} \mathrm{(Cat} \mathrm{\# H-3301;}$ Vector) in the microwave at full power. The slides were cooled to room temperature and then blocked with 5\% normal donkey serum (Cat \#D9663; Sigma-Aldrich) in phosphate buffered saline (PBS, Cat \#PBS01-02; Bioland Scientific) or TBS (Cat \#TBS03-02; Bioland Scientific) with 0.1\% Tween (PBS-T, Cat \#9005-64-5; Amresco) for $30 \mathrm{~min}$. hTESp slides were incubated for $8 \mathrm{~h}$ at $4^{\circ} \mathrm{C}$ at $1: 100$ with primary antibodies lamin (Cat \#108595; Abcam), PCNA (Cat \#sc-9857; Santa Cruz Biotechnology), CD4 (Cat \#MAS12259; Invitrogen), and CD11c (Cat \#ab11029; Abcam) in a humidified chamber. mTESp slides were also incubated for $8 \mathrm{~h}$ at $4^{\circ} \mathrm{C}$ at $1: 100$ with primary antibodies to green fluorescent protein (GFP, Cat \# 290; Abcam), PCNA, CD4, and CD11c. After $8 \mathrm{~h}$, slides were washed in PBS-T or TBS-T (for CD4 and CD11c) and incubated in secondary antibodies Alexa Fluor 488 (Cat \#705-546-147; Jackson Immunoresearch) or indocarbocyanine3 (Cy3, Cat \#712-165150; Jackson Immunoresearch) at 1:200 at room temperature for $1 \mathrm{~h}$. Slides were rinsed with PBS-T and mounted in Vectashield with DAPI (Cat \#H-1200; Vector). Images were taken with a fluorescent microscope at $40 \times($ Leica DM1600B). All hTESp slides were analyzed for costaining with lamin to confirm human origin whereas mTESp slides were analyzed for costaining with GFP to confirm Actin ${ }^{G F P}$ mouse spleen origin.

\section{Statistical analysis}

The mean number of Howell-Jolly bodies and target cells per hpf were compared between all groups: no surgery, splenectomy alone, splenectomy with mTESp, and splenectomy with hTESp, with one-way analysis of variance. All statistical analyses were performed with Graphpad Prism Software (Graphpad, San Diego, CA). Statistical significance was defined as $p<0.05$. 


\section{Results}

TESp can be generated from mouse and human spleen $\mathrm{OU}$

Mouse and human spleen OU were generated and evaluated with brightfield microscopy to confirm the presence of characteristic multicellular clusters (Fig. 1A), which demonstrated green fluorescence in the case of mouse spleen OU derived from Actin ${ }^{G F P}$ mouse spleen (Fig. 1D). Hematoxylin and eosins of human spleen OU before implantation also demonstrate multicellular clusters composed of multiple cell types (Supplementary Fig. S1A). Immunofluorescent (IF) staining of preimplantation human spleen OU demonstrates PCNA-positive cells, indicating the presence of proliferating cells in the implanted OU (Supplementary Fig. S1B). The spleen OU were then seeded onto polymers for omental implantation (Fig. 1A inset). After 4 weeks, both hTESp and mTESp had formed as $5-10 \mathrm{~mm}$ reddish brown tissues with vascular ingrowth. (Fig. 1B, E). TESp explants for both mouse- and human-derived donor cells demonstrated cellular architecture that resembles the red pulp, white pulp, and trabeculae that construct the normal architecture of native spleen (Fig. 1C, F).

\section{hTESp and mTESP bear proliferating human and murine cells}

There are proliferating cells of human origin within hTESp identified with lamin and PCNA (Fig. 2A). Not all human donor cells are proliferative and, vice versa, there are PCNA-positive cells that are not positive for the nuclear stain lamin, although the nucleus in some cases may be out of field, or these may indicate donor-derived cells that have differentiated. IF staining of mTESp with GFP and PCNA also demonstrated multiple instances of positive costaining, indicating proliferative donor-derived cells (Fig. 2B). These cells were located in regions similar to both the red and white pulps of native spleen.
FIG. 1. Histology of preimplantation spleen OU, gross appearance of TESp, and histology of subsequent mTESp and hTESp. Brightfield imaging of human spleen $\mathrm{OU}$ demonstrates multicellular clusters characteristic of OU with inset depicting preimplantation scaffolds loaded with human spleen OU (A). At 4 weeks, hTESp is $\sim 5 \mathrm{~mm}$ without obvious visible polymer (B). H\&E of hTESP demonstrates characteristic splenic architecture including white pulp surrounded by trabeculae, indicated by white and blue arrows, respectively (C). Fluorescent imaging of murine spleen OU from GFP donor mice with characteristic multicellular clusters displaying green fluorescence (D). After 4 weeks, mTESp explant is $\sim 10 \mathrm{~mm}$ without obvious remaining polymer $(\mathbf{E})$. H\&E of mTESp with components of normal spleen architecture including red pulp, indicated by the red arrow $(\mathbf{F})$. All scale bars $=50 \mu \mathrm{m}$. GFP, green fluorescent protein; H\&E, hematoxylin and eosin; hTESp, human tissue-engineered spleen; mTESp, murine tissue-engineered spleen; OU, organoid units; TESp, tissueengineered spleen. Color images are available online.
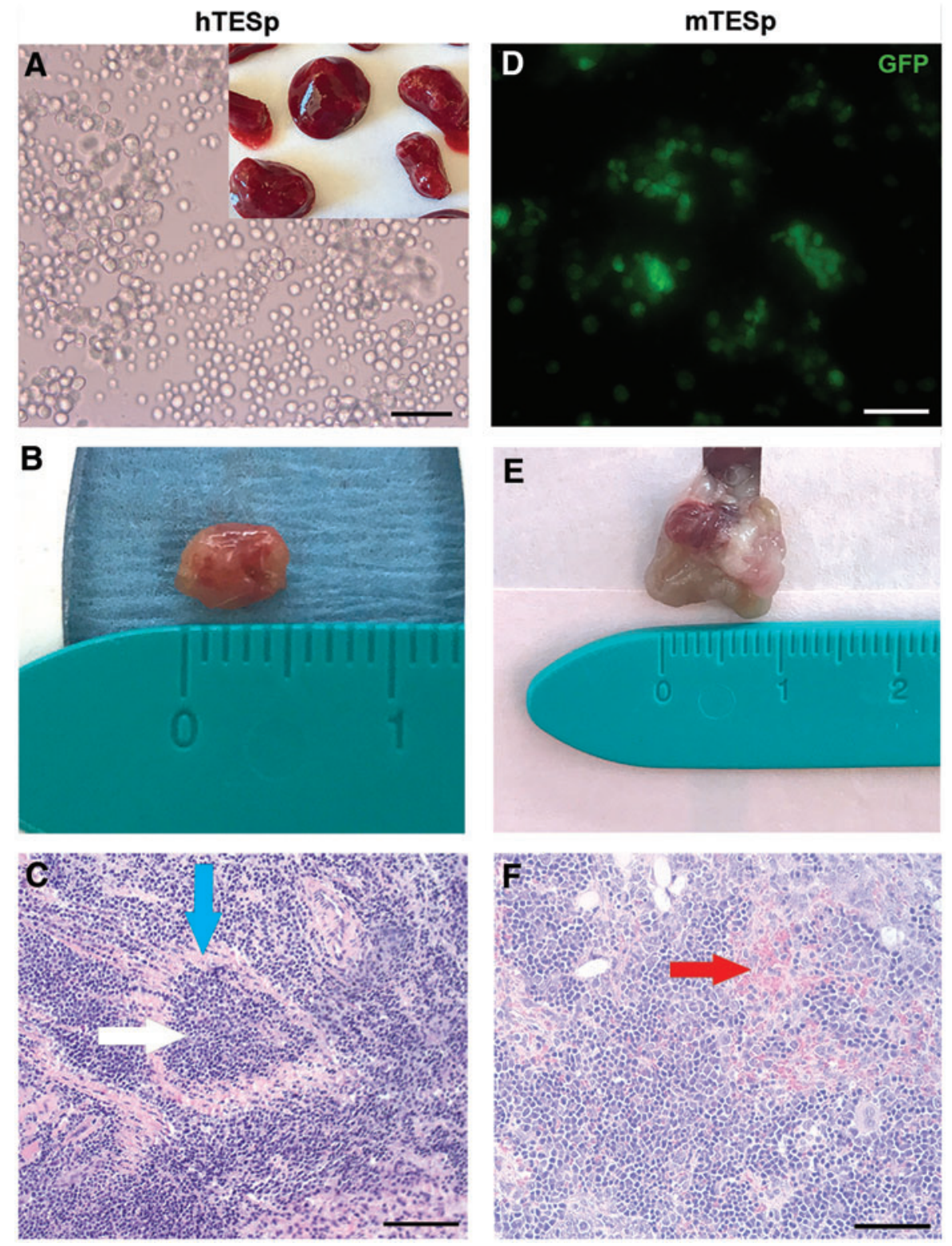
A

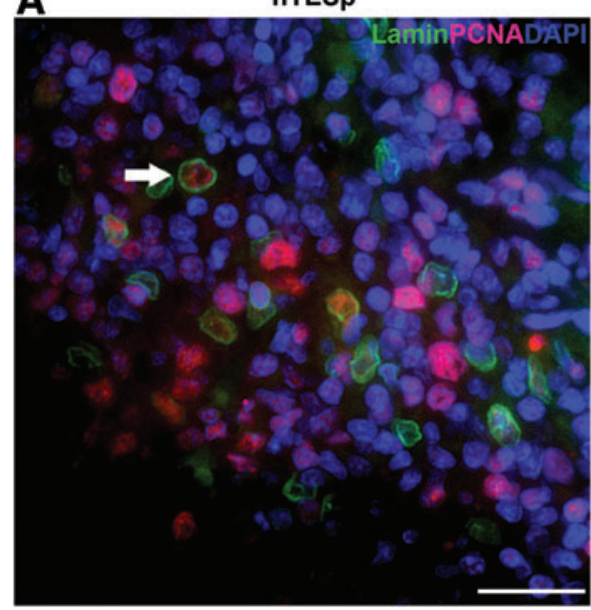

B

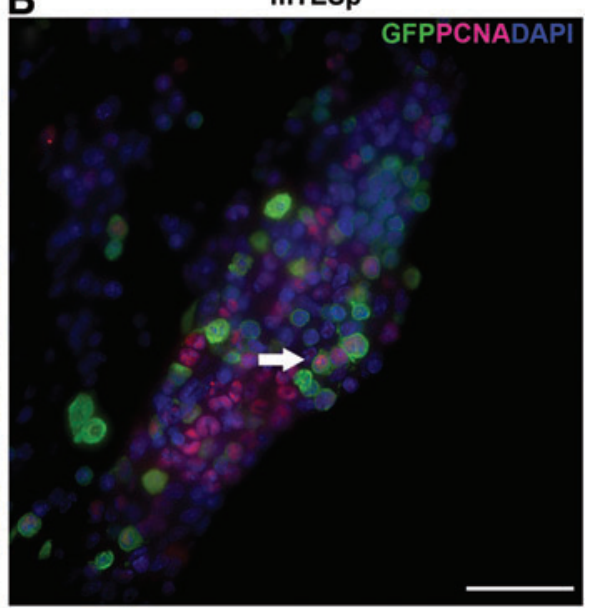

FIG. 2. IF staining of TESp to evaluate for markers of cellular proliferation and donor cell origin. IF imaging of hTESp with positive costaining for the nuclear envelope stain lamin (green) and nuclear stain PCNA (red) indicated by the white arrow (A). IF imaging of mTESp with cells positively costaining for cytoplasmic GFP (green) and PCNA (red) indicated by the white arrow $(\mathbf{B})$. All scale bars $=$ $50 \mu \mathrm{m}$. IF, immunofluorescent; PCNA, proliferating cell nuclear antigen. Color images are available online.

\section{Postsplenectomy scavenging of damaged erythrocytes is restored by hTESp and mTESp at 1 month}

Blood smears from the following four groups of mice were analyzed for the presence of target cells and Howell-Jolly bodies: no surgery control (Fig. 3A), splenectomy alone (Fig. 3B), splenectomy + hTESp (Fig. 3C), and splenectomy + mTESp (Fig. 3D). Target cells are erythrocyte inclusions identified in damaged cells that are normally cleared if a functional spleen is present (Fig. 3A inset). Similarly, Howell-Jolly bodies are basophilic nuclear remnants in erythrocytes that are normally cleared by a functional spleen (Fig. 3B inset). There was no difference in the number of target cells or the platelet count between any of the groups (Fig. 4A, C). However, mice with splenectomy alone had significantly more Howell-Jolly bodies present than mice with no surgery (1.55 \pm 0.2 vs. $0.5 \pm 0.5, p=0.0075)$, splenectomy + mTESp (1.55 \pm 0.2 vs.
$0.64 \pm 0.35, p=0.0092)$, or mice with splenectomy + hTESp $(1.55 \pm 0.2$ vs. $0.375 \pm 0.2, p=0.0021)$ (Fig. 4B). The blood smears also contained both myeloid and lymphoid subpopulations of cells. Blood smears from mice with TESp implants demonstrated thrombocytes, lymphocytes, and myeloblast populations including neutrophils and basophils. From the blood smears alone, it was not readily apparent whether these lymphoid subpopulations were of host or TESp origin so additional staining was carried out.

\section{hTESp and mTESp have functional immune cells of human and mouse origin, respectively}

Sections of mTESp and hTESp as well as human and GFP mouse spleen were stained for CD4, a glycoprotein found on the surface of T cells, and CD11c that is highly expressed on
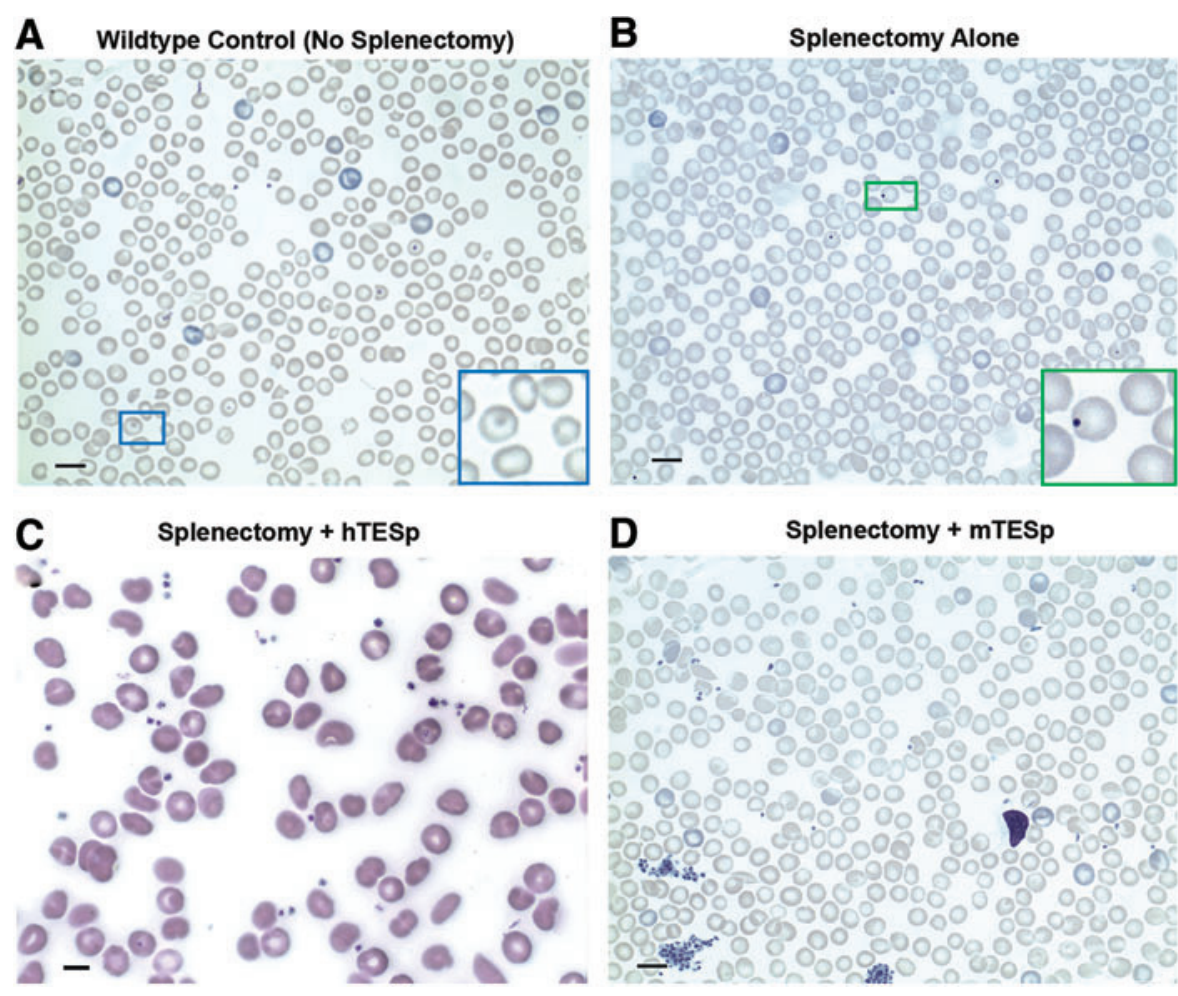

FIG. 3. Blood smear analysis from wildtype control with no surgical intervention (A), splenectomy alone (B), splenectomy + hTESp (C) and splenectomy + mTESp (D) to evaluate for presence of HowellJolly bodies and target cells as markers of asplenia. Inset in (A) indicates a target cell and inset in (B) a Howell-Jolly body. All scale bars $=10 \mu \mathrm{m}$. Color images are available online. 
A

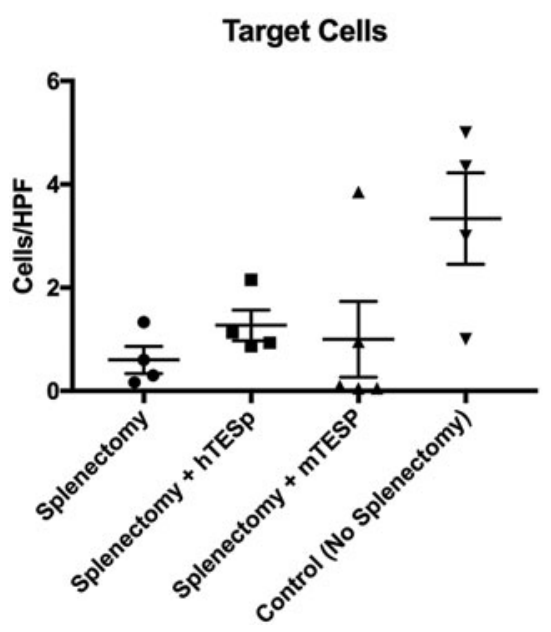

B

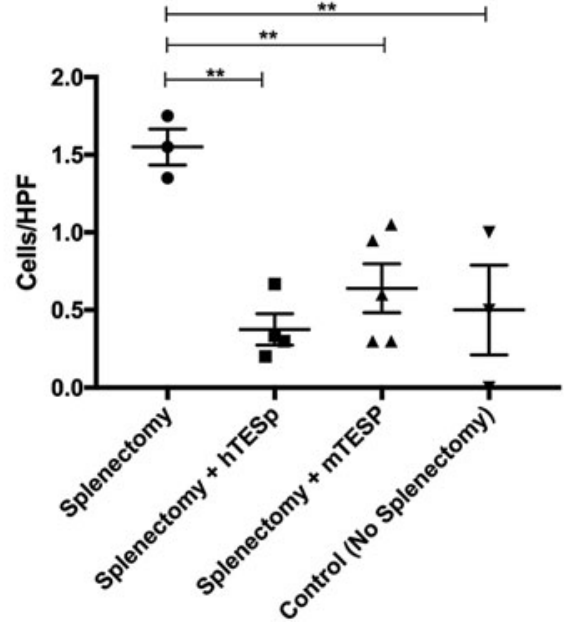

C

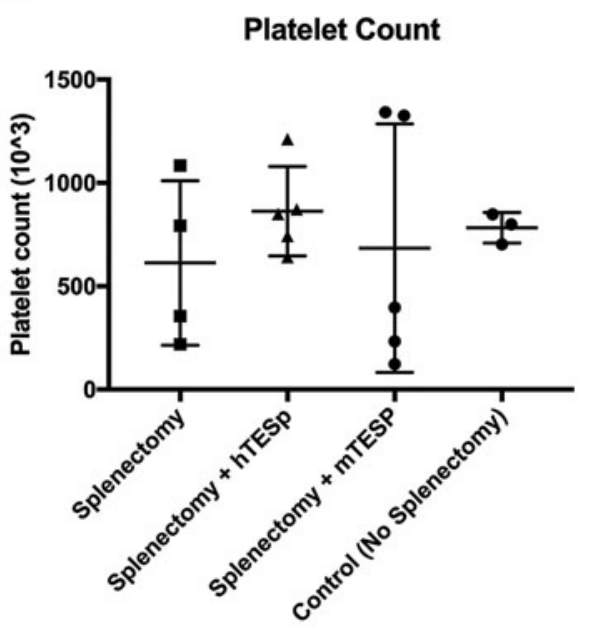

FIG. 4. One-way analysis of variance statistical analysis comparing number of target cells per hpf in each experimental group (A), number of Howell-Jolly bodies per hpf (B), and the platelet count (C) with significantly fewer Howell-Jolly bodies in mice implanted with TESp compared with mice with splenectomy alone, and no difference in number of target cells or platelet count. $* * p<0.01$. hpf, high-power field.

dendritic cells. mTESp sections were also stained for GFP to confirm donor cell origin, and hTESp sections were costained for lamin. hTESp had numerous lamin-positive cells (Fig. 5C, G), indicating cells of human origin, and some of these lamin-positive cells also had positive staining for CD4 (Fig. 5C) and CD11c (Fig. 5G). Similarly, mTESp also contained GFP-positive cells that costained with CD4 (Fig. 5D) and CD11c (Fig. 5H). Representative images of Actin $^{G F P}$ mouse and human splenic CD4 (Fig. 5A, B) and CD11c staining (Fig. 5E, F) are included for comparison.

\section{Discussion}

With alterations of a previous protocol, both mouse and human spleen OU could be generated from donor tissue, and after implantation, mTESp and hTESp formed and recapitulated rudimentary spleen architecture of zonation with trabeculae. The donor cell origin, whether human or mouse, was confirmed. The TESp contained PCNA-positive cells within the tissue at 4 weeks, indicating ongoing proliferative capacity of the donor cells (Fig. 2). Although not all TESp
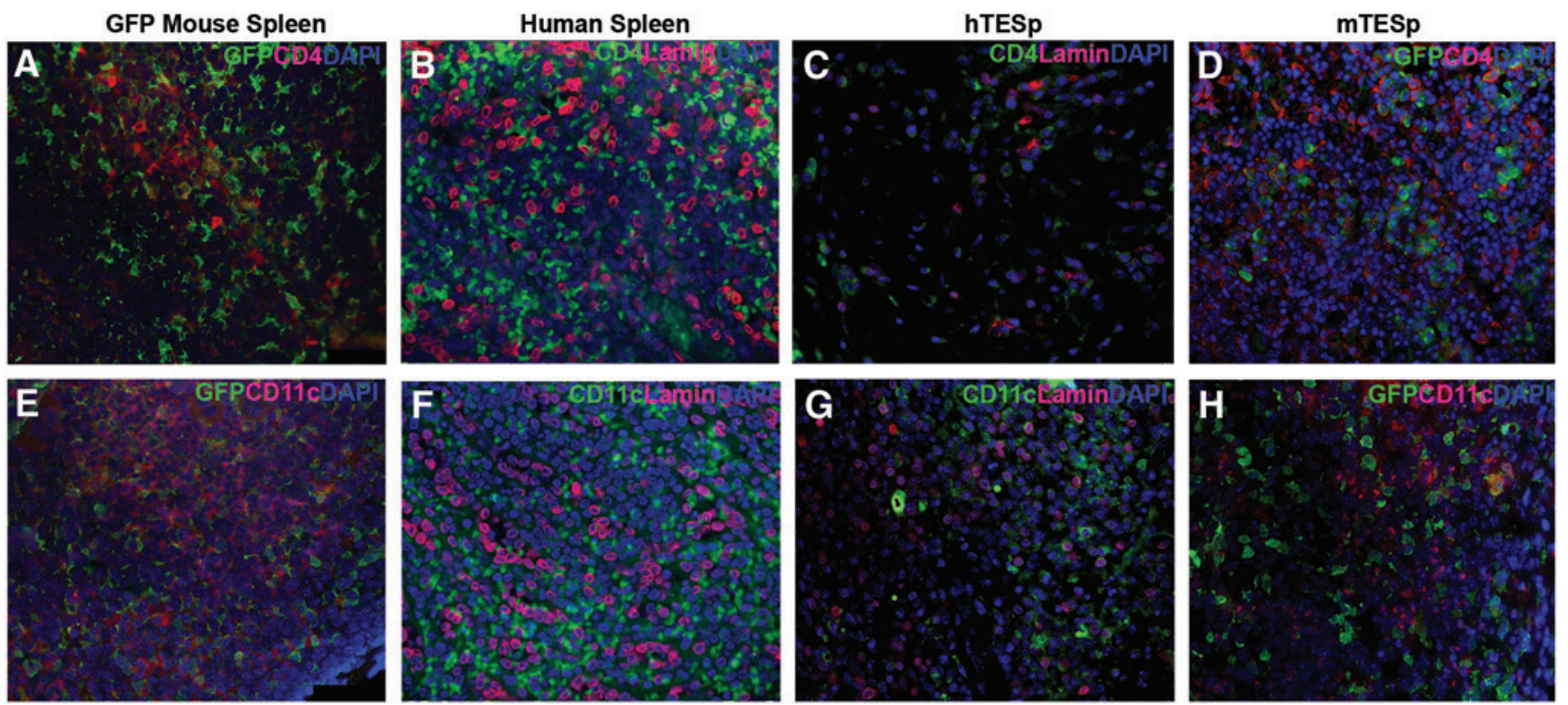

FIG. 5. IF staining to detect immune cells of donor origin required for spleen function. IF imaging of control Actin ${ }^{G F P}$ mouse and human spleen depicting GFP (green) or lamin (red) positive cells with cytoplasmic CD4 (A, B) or cytoplasmic CD11c (E, F) costaining for reference. hTESp stained for lamin and CD4 (C) as well as lamin and CD11c (G) with white arrows indicating copositive cells. mTESp stained for GFP and CD4 (D) and GFP and CD11c (H) with white arrows indicating copositive cells. All scale bars $=50 \mu \mathrm{m}$. Color images are available online. 
cells were identified as either lamin positive or GFP positive, staining of native controls (Fig. 5) shows parsimonious donor origin staining as well. After identifying rudimentary appropriate regional architecture in TESp and that this derived from proliferative donor cells, we investigated whether damaged erythrocytes are removed from the blood stream in animals with removal of the native spleen and only TESp to convey spleen function.

After splenectomy, damaged erythrocytes are scavenged in animals with splenectomy and either hTESp or mTESp at 1 month, as determined by blood smear analysis detecting the basophilic nuclear remnants in erythrocytes called Howell-Jolly bodies (Fig. 4), which were significantly increased in mice with a splenectomy without rescuing TESp compared with control mice or mice with TESp of either human or mouse origin. We did not know whether hTESp could clear damaged mouse erythrocytes, but postulated it might. It is known that without residual spleen, Howell-Jolly bodies persist indefinitely. ${ }^{19}$ In a study in male Wistar rats, Howell-Jolly bodies were no longer identified on blood smear when $22.5 \%$ of spleen mass was present. ${ }^{19}$

Both mTESp and hTESp contained CD4- and CD11cpositive cells that also indicated donor origin with either lamin or GFP costaining. Although there are multiple cell types of myeloid and lymphoid lineage required for the function of innate and adaptive immunity, both $\mathrm{T}$ cells and dendritic cells are required for spleen function. T cells in the spleen, marked by CD4, have further subsets of naive, memory, and effector $\mathrm{T}$ cells and are fewer in number than B cells. ${ }^{20}$ Further evaluation of these subsets, particularly in hTESp, may be helpful but also might be patient-specific and thus difficult to generalize. Dendritic cells in the spleen (CD11c) are antigen-presenting cells that capture antigens and, after processing, present these antigens to naive $T$ cells. Both partners in this exchange are present in TESp, whether derived from mouse or human donor cells.

Patients require splenectomy for a variety of reasons including trauma and hematologic disorders, yet although the spleen is not qualified as a vital organ, the loss of splenic function can result in devastating morbidities including death from OPSI, and perhaps later thrombotic events such as strokes, pulmonary hypertension, and myocardial infarctions since damaged erythrocytes are not readily cleared. ${ }^{21,22}$ Partial splenectomies have been performed in inherited red cell membrane disorders, such as the two most common hereditary spherocytosis and elliptocytosis, ${ }^{23}$ to retain spleen mass because spleen function is so important. In these conditions, it is no longer an advantage for the spleen to consume irregular erythrocytes because normal erythrocytes cannot be produced. ${ }^{24}$

Yet estimating the optimal splenic remnant to reduce erythrocyte loss and retain immune function may vary and is also dependent on retaining adequate blood flow. ${ }^{25}$ Similarly, partial splenectomy is considered in cases of tumor, vascular malformation, cyst, or other conditions in which some normal spleen might be safely retained. In partial versus total splenectomy, bleeding risks are higher because more vasculature and well-vascularized tissue must be divided. In cases of emergent spleen removal or partial splenectomy, in which estimates of remnant spleen are difficult, resupplying patients with autologous TESp might be of use. We have previously shown that various OU may be stored with vitrification, or conversion to a glass-like state, ${ }^{26}$ in case of the need for staged procedures. These data obtained from mouse and human donor cells indicate that spleen OU and the resulting TESp that forms in vivo might provide functional spleen tissue for patients in whom all or a portion of the spleen must be removed.

\section{Conclusion}

Mouse and human spleen OU generate TESp that is regionalized and proliferative. Both mTESp and hTESp reduce Howell-Jolly bodies in the peripheral blood and demonstrate cells of $\mathrm{T}$ and dendritic cell origin after 1 month in vivo. Because TESp could be a possible therapeutic option after emergent splenectomy or as a salvage for partial splenectomy, further investigation might allow for future human therapies.

\section{Disclosure Statement}

No competing financial interests exist.

\section{Author Contributions}

T.C.G. conceived and designed the study M.A.I., A.F., K.G., K.M.M., S.M.Z., A.I.S., K.L.F., and L.M.N acquired data. M.A.I., K.M.M., K.G., A.F., S.M.Z., and T.C.G. analyzed and interpreted data. M.A.I., K.M.M., K.G., and S.M.Z. drafted the article. T.C.G critically revised the article.

\section{Funding Information}

This project was unfunded.

\section{Supplementary Material}

Supplementary Figure S1

\section{References}

1. Di Sabatino, A., Carsetti, R., and Corazza, G.R. Postsplenectomy and hyposplenic states. Lancet 378, 86, 2011.

2. Iolascon, A., Andolfo, I., Barcellini, W., et al. Recommendations regarding splenectomy in hereditary hemolytic anemias. Haematologica 102, 1304, 2017.

3. Carlstedt, A., and Tholin, B. Infectious complications after splenectomy. Acta Chir Scand 150, 607, 1984.

4. Shaw, J.H., and Print, C.G. Postsplenectomy sepsis. Br J Surg 76, 1074, 1989.

5. Krivit, W., Giebink, G.S., and Leonard, A. Overwhelming postplenectomy infection. Surg Clin North Am 59, 223, 1979.

6. Velanovich, V., and Tapper, D. Decision analysis in children with blunt splenic trauma: the effects of observation, splenorrhaphy, or splenectomy on quality-adjusted life expectancy. J Pediatr Surg 28, 179, 1993.

7. Shatney, C.H. Complications of splenectomy. Acta Anaesthesiol Belg 38, 333, 1987.

8. Mebius, R.E., and Kraal, G. Structure and function of the spleen. Nat Rev Immunol 5, 606, 2005.

9. Cesta, M.F. Normal structure, function, and histology of the spleen. Toxicol Pathol 34, 455, 2006.

10. Grikscheit, T.C., Sala, F.G., Ogilvie, J., et al. Tissueengineered spleen protects against overwhelming pneumococcal sepsis in a rodent model. J Surg Res 149, 214, 2008.

11. Barthel, E.R., Speer, A.L., Levin, D.E., et al. Tissue engineering of the intestine in a murine model. J Vis Exp e4279, 2012. 
12. Sala, F.G., Matthews, J.A., Speer, A.L., Torashima, Y., Barthel, E.R., and Grikscheit, T.C. A multicellular approach forms a significant amount of tissue-engineered small intestine in the mouse. Tissue Eng Part A 17, 1841, 2011.

13. Sala, F.G., Kunisaki, S.M., Ochoa, E.R., Vacanti, J., and Grikscheit, T.C. Tissue-engineered small intestine and stomach form from autologous tissue in a preclinical large animal model. J Surg Res 156, 205, 2009.

14. Spurrier, R.G., Speer, A.L., Hou, X., El-Nachef, W.N., and Grikscheit, T.C. Murine and human tissue-engineered esophagus form from sufficient stem/progenitor cells and do not require microdesigned biomaterials. Tissue Eng Part A 21, 906, 2015.

15. Grikscheit, T.C., Ochoa, E.R., Ramsanahie, A., et al. Tissue-engineered large intestine resembles native colon with appropriate in vitro physiology and architecture. Ann Surg 238, 35, 2003.

16. Grant, C.N., Mojica, S.G., Sala, F.G., et al. Human and mouse tissue-engineered small intestine both demonstrate digestive and absorptive function. Am J Physiol Gastrointest Liver Physiol 308, G664, 2015.

17. Speer, A.L., Sala, F.G., Matthews, J.A., and Grikscheit, T.C. Murine tissue-engineered stomach demonstrates epithelial differentiation. J Surg Res 171, 6, 2011.

18. Barthel, E.R., Levin, D.E., Speer, A.L., et al. Human tissueengineered colon forms from postnatal progenitor cells: an in vivo murine model. Regen Med 7, 807, 2012.

19. Marques, R.G., Lucena, S.B., Caetano, C.E., de Sousa, V.O., Portela, M.C., and Petroianu, A. Blood clearance of Howell-Jolly bodies in an experimental autogenic splenic implant model. Br J Surg 101, 820, 2014.

20. Langeveld, M., Gamadia, L.E., and ten Berge, I.J. Tlymphocyte subset distribution in human spleen. Eur J Clin Invest 36, 250, 2006.
21. Schilling, R.F. Spherocytosis, splenectomy, strokes, and heat attacks. Lancet 350, 1677, 1997.

22. Hoeper, M.M., Niedermeyer, J., Hoffmeyer, F., Flemming, P., and Fabel, H. Pulmonary hypertension after splenectomy? Ann Intern Med 130, 506, 1999.

23. Da Costa, L., Galimand, J., Fenneteau, O., and Mohandas, N. Hereditary spherocytosis, elliptocytosis, and other red cell membrane disorders. Blood Rev 27, 167, 2013.

24. Madenci, A.L., Armstrong, L.B., Kwon, N.K., et al. Incidence and risk factors for sepsis after childhood splenectomy. J Pediatr Surg 54, 1445, 2019.

25. Bader-Meunier, B., Gauthier, F., Archambaud, F., et al. Long-term evaluation of the beneficial effect of subtotal splenectomy for management of hereditary spherocytosis. Blood 97, 399, 2001.

26. Spurrier, R.G., Speer, A.L., Grant, C.N., Levin, D.E., and Grikscheit, T.C. Vitrification preserves murine and human donor cells for generation of tissue-engineered intestine. J Surg Res 190, 399, 2014.

Address correspondence to: Tracy C. Grikscheit, $M D$ Developmental Biology and Regenerative Medicine Saban Research Institute Children's Hospital Los Angeles 4650 Sunset Boulevard Los Angeles, CA 90027

E-mail: tgrikscheit@chla.usc.edu

Received: July 12, 2019

Accepted: October 31, 2019

Online Publication Date: December 12, 2019 